\title{
Stress Distribution in Maxillary Central Incisors without Ferrules: A Finite Element Analysis of Post and Core Systems
}

\author{
Akihiro Tagahara, Chihiro Masaki, Tomotaka Nodai, Takashi Munemasa, Taro Mukaibo, \\ Yusuke Kondo, Ryuji Hosokawa
}

Division of Oral Reconstruction and Rehabilitation, Kyushu Dental University, Kitakyushu, Japan

Email: masaki@kyu-dent.ac.jp

How to cite this paper: Tagahara, A., Masaki, C., Nodai, T., Munemasa, T., Mukaibo, T., Kondo, Y. and Hosokawa, R. (2021) Stress Distribution in Maxillary Central Incisors without Ferrules: A Finite Element Analysis of Post and Core Systems. Open Journal of Stomatology, 11, 251-262. https://doi.org/10.4236/ojst.2021.117022

Received: June 12, 2021

Accepted: July 11, 2021

Published: July 14, 2021

Copyright $\odot 2021$ by author(s) and Scientific Research Publishing Inc. This work is licensed under the Creative Commons Attribution International License (CC BY 4.0).

http://creativecommons.org/licenses/by/4.0/

(c) (i) Open Access

\begin{abstract}
Purpose: The purpose of this study was to identify optimal post and core materials for central incisors without ferrules using three-dimensional finite element analysis and three-point bending tests. Methods: Stress analyses were performed with six models: cast metal post and core (MP), composite resin core alone, straight fiber-reinforced post-composite resin core (FSR), tapered fiber-reinforced post-composite resin core, straight titanium postcomposite resin core (TSR), and tapered titanium post-composite resin core (TTR). A $100-\mathrm{N}$ load was applied to the lingual surface at a $45^{\circ}$ angle to the long axis of the tooth. Maximum von Mises stress distributions were calculated with finite element analysis software. Five samples each of composite resin, straight fiber-reinforced post, straight titanium post, straight fiberreinforced post and composite resin, and straight titanium post and composite resin were subjected to three-point bending tests, followed by analysis of variance and Tukey's multiple comparison test. Results: Stress distribution was optimal on TTR. Maximum von Mises stress on the cervical side of the post was greatest in TSR (693 MPa) and TTR (556 MPa). Maximum stress on the apical side of the post was greatest in MP (110 MPa). Maximum stress in surrounding dentin was lowest in MP (203 MPa) and TTR (250 MPa). Gap distance was smallest in MP $(0.09 \mathrm{~mm})$ and largest in FSR $(0.26 \mathrm{~mm})$. Mean maximum three-point bending force was lowest in composite resin $(26.9 \mathrm{~N} / \mathrm{mm})$ and highest in titanium post and composite resin $(97.1 \mathrm{~N} / \mathrm{mm})$. Titanium post bending strength was consistently greater than that of the fiber-reinforced post $(p<0.01)$. Conclusion: These results revealed optimal stress distribution and high bending strength with the tapered titanium post and resin combination, suggesting that this combination can most effectively prevent root or post fracture in an anterior tooth without a ferrule.
\end{abstract}




\section{Keywords}

Fiber Post, Titanium Post, Ferrule, Three-Dimensional Finite Element Analysis, Three-Point Bending Test

\section{Introduction}

Tooth loss because of dental caries and periodontal disease has decreased in accordance with the increasing promotion of dental prevention and maintenance care approaches. Root fracture in devitalized teeth has thus become one of the leading causes of extraction [1]. Metal posts and cores have long been used to treat devitalized teeth; however, the risk of root fracture because of a higher elastic modulus (compared with dentin) has frequently been reported [2] [3] [4]. Composite resin cores with prefabricated posts are increasingly used to reduce the risk of root fracture because the elastic modulus of composite resin cores is lower than that of metal cores. High-strength stainless-steel metal posts have been used as prefabricated posts [2] [3]; however, they have limitations such as poor esthetics due to the metal color, as well as a greater elastic modulus than that of either dentin or composite resin. Therefore, glass fiber-reinforced composite posts are now frequently used. These posts are advantageous because their modulus of elasticity is similar to that of dentin and comparable with that of metal posts and cores; moreover, they exhibit white semitransparency and do not cause esthetic interference with the crown prosthesis (typically encountered in the use of ceramics) [4]. In an in vitro fatigue test with repeated loading, root fracture occurred with a cast metal post and core, but not with a composite resin core used with a metal post or glass fiber post [5]. Furthermore, in a 15-year retrospective clinical study, the incidence of root fracture was significantly greater in teeth with metal cores than in those with composite resin cores [6].

Previous studies have shown relationships between root fracture and post and core materials, as well as between root fracture and ferrule status (presence or absence) [7] [8] [9]. Because resistance to wedge-like loads and torque transmitted through a post can be achieved by securing a ferrule [10], the application of an appropriate ferrule by orthodontic extrusion and surgical periodontal treatment is recommended for teeth without ferrules. Compared with vertical fracture, fracture and detachment of the post and tooth neck fracture occur more frequently in a glass fiber-reinforced post [11] [12]; therefore, the use of a glass fiber-reinforced post may reduce the risk of complications leading to extraction in teeth where posts are secured by ferrules.

Retreatment is challenging in teeth without ferrules because the supracrestal tissue attachment cannot be secured in vertical root fractures or in fractures of the coronal region of the root, which are indicated for extraction. Therefore, for severely damaged teeth without ferrules, appropriate posts and cores differ from those used in teeth with ferrules. In this study, three-dimensional finite element 
analysis and three-point bending tests were performed with various post and core systems to identify optimal post and core materials for central incisors without ferrules. The null hypothesis was that the combination of titanium post and composite resin would not be effective for preventing root fracture and post fracture in central incisors without ferrules.

\section{Material and Methods}

In the three-dimensional finite element analysis, stress analyses were performed with six models: a cast metal post and core (MP), a composite resin core without a post (RP), a straight fiber-reinforced post and composite resin core (FSR), a tapered fiber-reinforced post and composite resin core (FTR), straight titanium post-composite resin (TSR), and tapered titanium post-composite resin (TTR).

DICOM data (KaVo 3D eXam; resolution: $200 \mu \mathrm{m}$, tube voltage: $120 \mathrm{kV}$, tube current: $5 \mathrm{~mA}$, acquisition duration: $7 \mathrm{~s}$ ) of a maxillary central incisor without caries acquired for orthodontic treatment from a teenage female patient were converted to Standard Triangle Language data with Invivo 5.3 Medical Design Studio (KaVo, Biberach, Germany). A three-dimensional model of this tooth with the periodontal ligament, cortical bone, cancellous bone, and gingiva was constructed based on these data with Geomagic Freeform Plus V2019.2.50 64-bit (3D SYSMEMS, South Carolina, USA).

Gutta-percha was retained $(5 \mathrm{~mm})$ in the apical region, the post length was set at two-thirds of the root canal, and the thickness was set at a $1 \mathrm{~mm}$ diameter at the tip of the post. Straight and tapered posts were designed with diameters of 1 $\mathrm{mm}$ each, with $2^{\circ}$ of tapering. The ferrule was set at $0 \mathrm{~mm}$ and the crown was repaired with IPS e.max Press (lithium disilicate) in the models (Figure 1(a) and Figure 1(b)).

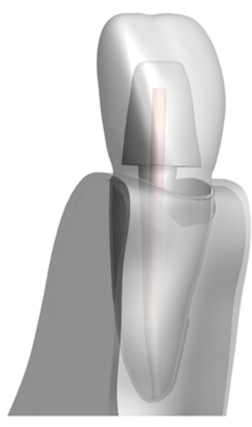

(a)

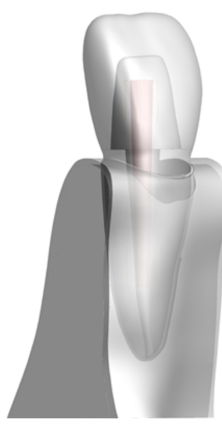

(b)

Figure 1. Six distinct post and core materials were simulated for central incisors without ferrules. Gutta-percha was left $(5 \mathrm{~mm})$ in apical region, post length was set at two-thirds of root canal, and thickness was set at $1 \mathrm{~mm}$ diameter at tip of post. Straight and tapered posts were designed with diameters of $1 \mathrm{~mm}$ each, with $2^{\circ}$ of tapering. Ferrule was set at 0 $\mathrm{mm}$ and crown was repaired with IPS e.max Press (lithium disilicate) in models (a) straight fiber-reinforced post and composite resin core (FSR) and straight titanium postcomposite resin (TSR) or (b) tapered fiber-reinforced post and composite resin core (FTR) and tapered titanium post-composite resin (TTR). Load of $100 \mathrm{~N}$ was applied to center of lingual surface at $45^{\circ}$ angle to long axis of tooth. 
The models constructed with Geomagic Freeform Plus were output in SOLIDWORKS (R) 2018 x64 Edition SP3.0 (Dassault Systèmes SolidWorks Corporation) of analysis software, followed by element division (mesh). The total number of nodal points was 91,088 , while the total number of nodal elements was 52,507 . The characteristics of all materials in the models are shown in Table 1 [13]-[18]. Under the constraint condition, the post and core were adhered to the crown; they were in contact with dentin, but did not adhere to it.

A load of $100 \mathrm{~N}$ was applied to the center of the lingual surface at an angle of $45^{\circ}$ to the long axis of the tooth. Maximum von Mises stress distributions in the post (cervical and apical sides) and surrounding dentin for each model were calculated with the finite element analysis software SOLIDWORKS (R) 2018 x64 Edition SP3.0. The distance of the gap generated between dentin and the core material in the sagittal view was also measured.

The three-point bending test was performed to investigate the bending strengths of five types of test pieces: composite resin (resin), fiber-reinforced post (fiber), titanium post (titanium), fiber-reinforced post and composite resin (fiber/resin), and titanium post and composite resin (titanium/resin).Five fiber-reinforced posts (GC fiber post) (diameter: $1.0 \mathrm{~mm}$, length: $14 \mathrm{~mm}$ ) and titanium posts (ParaPost XP, Coltene/Whaledent, Ohio, USA) (diameter: $1.0 \mathrm{~mm}$, length: 14 $\mathrm{mm}$ ) each were used for the respective fiber and titanium groups. Five test pieces each of fiber/resin and titanium/resin groups were prepared by placing fiber-reinforced and titanium posts in the center and designing a jig for composite resin (Clearfil DC core Automix one, Kuraray Noritake, Tokyo, Japan) adherence around them. Fiber posts were etched with phosphoric acid, washed with water, and dried; they were then pretreated with Ceramic primer (Clearfil Ceramic Primer Plus, Kuraray Noritake, Tokyo, Japan). Titanium posts were sand blasted at $0.15 \mathrm{MPa}$ from a distance of $10 \mathrm{~mm}$, then ultrasonically cleaned and dried for 10 minutes; they were then pretreated with Alloy Primer (Kuraray Noritake Dental, Inc.). The diameter was set to $1.8 \mathrm{~mm}$ and the length was set to

Table 1. Characteristics of all materials in models.

\begin{tabular}{lcccc}
\hline & Structure/Material & Elastic Modulus (MPa) & Poisson Ratio & Reference \\
\hline 1 & Dentin & 18,600 & 0.31 & {$[13]$} \\
2 & Ligament & 68.9 & 0.45 & {$[13]$} \\
3 & Cortical bone & 13,700 & 0.30 & {$[13]$} \\
4 & Cancellous bone & 1370 & 0.30 & {$[13]$} \\
5 & Gingiva & 19.6 & 0.30 & {$[13]$} \\
6 & Gutta-percha & 0.69 & 0.45 & {$[13]$} \\
7 & Gold alloy post & 95,000 & 0.33 & {$[14]$} \\
8 & Composite resin & 12,000 & 0.33 & {$[15]$} \\
9 & Glass fiber-reinforced composite posts & 40,000 & 0.26 & {$[16]$} \\
10 & Titanium post & 103,400 & 0.33 & {$[17]$} \\
11 & Crown (IPS e.max Press) & 82,300 & 0.22 & {$[18]$} \\
\hline
\end{tabular}


$14 \mathrm{~mm}$ in resin, fiber/resin, and titanium/resin groups. Sample size was determined with a power analysis to provide statistical significance $(\alpha=0.05)$ at $80 \%$ power, on the basis of previous findings [19] [20]. All measurements were obtained by investigators who were blinded to the experimental conditions.

The three-point bending test was performed at a distance between fulcrums of $10 \mathrm{~mm}$ and speed of $0.5 \mathrm{~mm} / \mathrm{s}$ with an Autograph AGS-X (Shimadzu Corporation, Kyoto, Japan). The maximum load representing the force that destroyed the test piece was applied to five types of test pieces for comparisons.

GraphPad Prism 5.0 for Mac OS X (GraphPad Software Inc., San Diego, CA, USA) was used to analyze the values in each group. All data in the three-point bending test were represented as the mean \pm standard deviation and were subjected to analysis of variance; the Tukey multiple comparison test was used for post hoc analysis. The level of significance was set at $\mathrm{p}<0.05$.

\section{Results}

The contour diagram in finite element analysis showed that strong stress was loaded on the entire MP over the apical side, whereas stress was concentrated on the cervical side of the post in RP, FSR, FTR, and TSR. The distribution on TTR was intermediate between the former and latter, showing the lowest stress concentration (Figure 2).

Maximum von Mises stress distribution on the cervical side of the post was greater in TSR (693 MPa) and TTR (556 MPa) than in MP (321 MP), RP (250 MP), FSR (296 MPa), or FTR (291 MPa) (Figure 3(a)). Furthermore, maximum

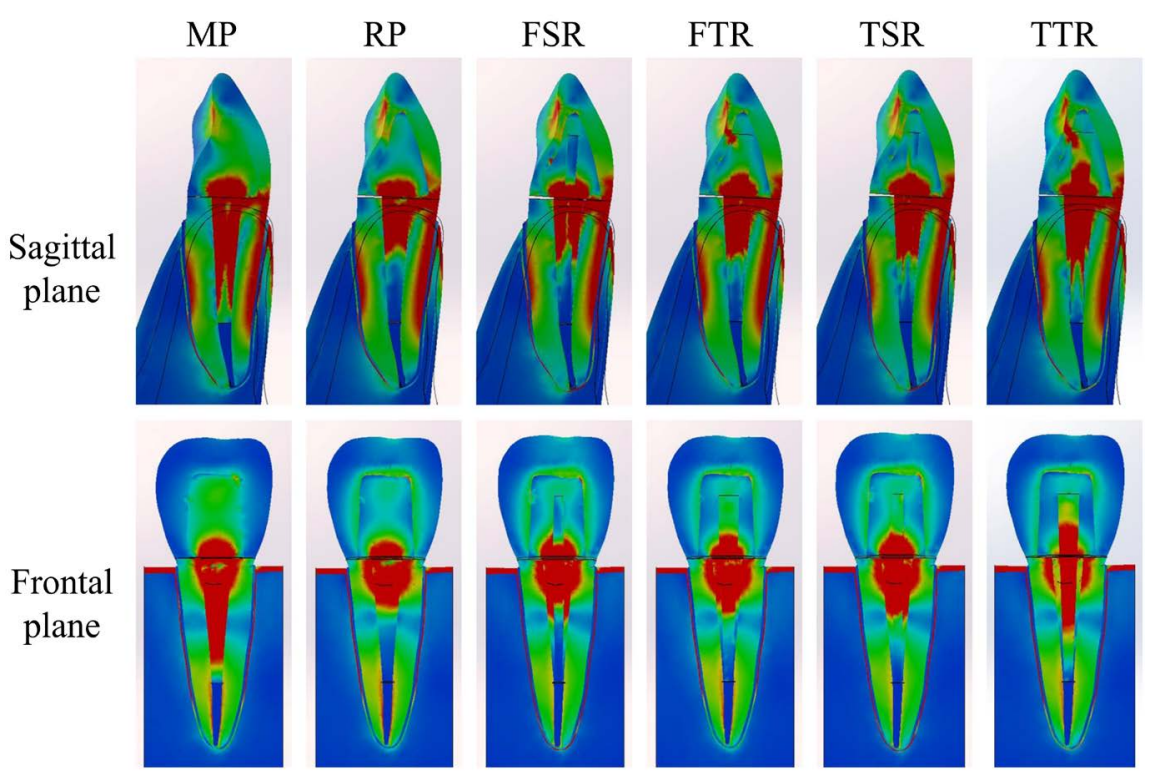

Figure 2. Contour diagram showing stress distributions for all models (sagittal and frontal planes). Abbreviations: MP, metal post and core; RP, composite resin core without post; FSR, straight fiber-reinforced post and composite resin core; FTR, tapered fiberreinforced post and composite resin core; TSR, straight titanium post-composite resin; TTR, tapered titanium post-composite resin. 


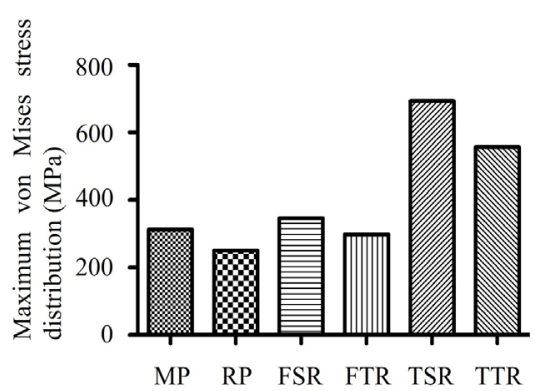

(a)

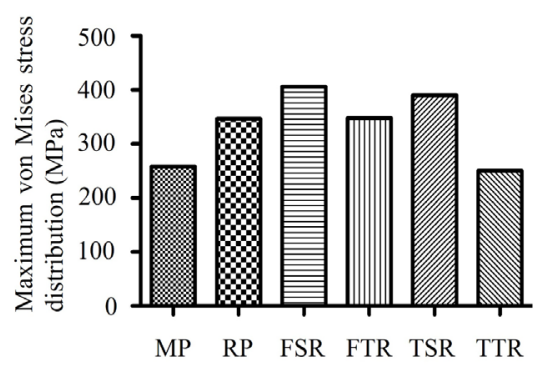

(c)

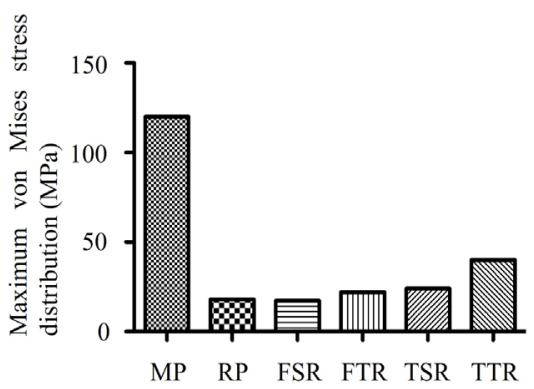

(b)

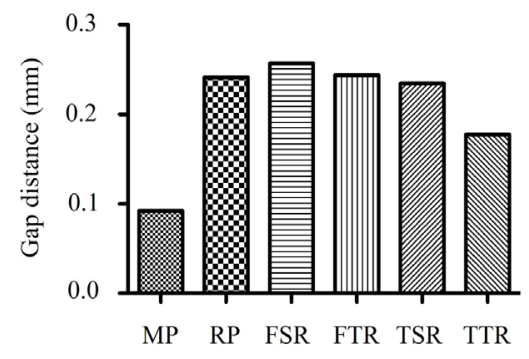

(d)

Figure 3. Maximum von Mises stress distributions for all models: (a) cervical side in post; (b) apical side in post, (c) in surrounding dentin; and (d) distance of gap generated between dentin and core material in sagittal view. Abbreviations: MP, metal post and core; $\mathrm{RP}$, composite resin core without post; FSR, straight fiber-reinforced post and composite resin core; FTR, tapered fiber-reinforced post and composite resin core; TSR, straight titanium post-composite resin; TTR, tapered titanium post-composite resin.

stress on the apical side of the post was greater in MP (110 MPa) than in RP (12 MPa), FSR (14 MPa), FTR (19 MPa), TSR (24 MPa), or TTR (40 MPa) (Figure $3(\mathrm{~b})$ ). Maximum stress in surrounding dentin was lower in MP (203 MPa) and TTR (250 MPa) than in RP (347 MPa), FSR (400 MPa), FTR (358 MPa), or TSR (390 MPa) (Figure 3(c)).

The gap distance was smallest in MP $(0.09 \mathrm{~mm})$. The largest gap $(0.26 \mathrm{~mm})$ was evident in FSR and was similar to the gaps in FTR $(0.24 \mathrm{~mm}), \mathrm{RP}(0.24$ $\mathrm{mm})$, and TSR $(0.23 \mathrm{~mm})$. The gap distance in TTR $(0.18 \mathrm{~mm})$ was between the distance evident in MP and the distances evident in FSR, FTR, RP, and TSR (Figure 3(d)).

Three-point bending caused fracture in the composite resin and fiber-reinforced post at a load near the maximum stress. In contrast, fracture did not occur due to plastic deformation in the titanium post, which is a ductile material. Therefore, the maximum load was calculated from the bending load-deflection curve at the proportional limit.

The results of the three-point bending test are shown in Figure 4. The mean maximum force of the resin was $26.86 \pm 1.91 \mathrm{~N}$, that of fiber was $51.45 \pm 1.11 \mathrm{~N}$, and that of titanium was $65.12 \pm 0.89 \mathrm{~N}$. Moreover, the mean maximum force of fiber/resin was $75.53 \pm 7.52 \mathrm{~N}$ and that of titanium/resin was $97.12 \pm 7.82 \mathrm{~N}$. Regardless of the presence or absence of composite resin, the bending strength 


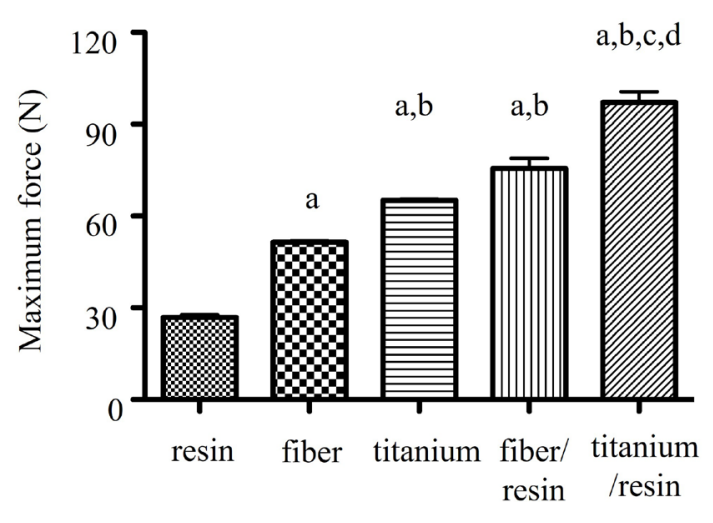

Figure 4. Three-point bending test revealed that maximum force loaded on fiber/resin was approximately 2.8 -fold greater than that on resin, while maximum force loaded on titanium/resin was approximately 1.3fold greater than that on fiber/resin. Data are expressed as mean \pm standard deviation. (a) $\mathrm{p}<0.01$ vs resin group, (b) $\mathrm{p}<0.01$ vs fiber group, (c) $\mathrm{p}<0.01$ vs titanium group, (d) $\mathrm{p}<0.01$ vs fiber/resin group $(\mathrm{n}=5)$.

of the titanium post was greater than that of the fiber-reinforced post $(\mathrm{p}<0.01)$. In addition, the maximum force loaded on fiber/resin was approximately 2.8 fold greater than that loaded on the resin, while the maximum force loaded on titanium/resin was approximately 1.3 -fold greater than that loaded on fiber/resin.

\section{Discussion}

The results of this study supported rejecting the null hypothesis. The results of the finite element analysis and three-point bending test indicated that the combination of the tapered titanium post and composite resin more effectively prevented fracture, compared with the glass fiber-reinforced post, in an anterior tooth without a ferrule.

In a three-dimensional finite element analysis, it is important to construct an analytical model that is similar to the approach used in actual clinical practice. Previous studies used a model with a thick post [21] [22] or a model that did not undergo flare preparation in accurate canal treatment [23]. In this study, flare preparation was performed in accordance with the principle of root canal treatment; post and core models were used that avoided excess post formation corresponding to the root canal. The results were markedly affected by the adherence condition of the model. In clinical practice, adhesion in the deep part of a root canal is difficult to achieve despite the application of adhesive resin cement to the post [24]. Moreover, long-term repeated loading destroys the adhesive interface, leading to complications that include detachment of the post and root fracture [5] [25]. Therefore, to simulate a state in which adhesive resin cement loses its adhesive strength due to repeated loading, a condition was used that did not involve the adhesion of dentin to the post and core. In previous finite element analysis studies, a contour diagram showing stress distribution was only 
evaluated in the sagittal plane. In this study, the contour diagram was compared in both the sagittal and frontal planes, which clearly revealed differences among the groups.

Notably, finite element analysis showed that stress was loaded on the entire MP to the post tip, whereas stress was only loaded on the shallow region of the post in RP, FSR, FTR, and TSR; these findings suggested that the risk of vertical fracture of the root was lower when using a composite resin core with a prefabricated post than when using a cast metal post. However, in the composite resin core with a prefabricated post (FSR, FTR, and TSR), high stress was loaded on dentin of the tooth neck, whereas less stress was loaded on the tapered titanium post. When horizontal fracture occurs in the tooth neck in teeth without ferrules, it is not possible to secure the supracrestal tissue attachment without performing extraction or crown lengthening surgery; thus, extraction may be indicated. A tapered titanium post may effectively reduce the risk of horizontal fracture in the tooth neck region.

The gap distance is related to resistance to detachment of the post and core, as well as the risk of secondary caries induced by the elution of cement. The risk of detachment decreases with smaller gap distances, which may prevent secondary caries. The results in this study showed that the gap distance in the metal post was significantly smaller than that of any other system; although cast metal posts are at a risk of root fracture, the elution of cement and crown detachment is presumably minimized, thereby preventing fracture of the post itself. In contrast, the gap distance was larger in the composite resin core with a prefabricated post than in MP; the smallest value among prefabricated posts was evident for the tapered titanium post, suggesting that detachment does not readily occur in tapered titanium posts, which may reduce the risk of secondary caries.

Importantly, when a parafunction such as bruxism is present, an excessive occlusal force is generated, thus increasing the risk of post fracture. Therefore, a post material with stronger bending strength should be selected to reduce this risk of post fracture. In the three-point bending test, the maximum forces of fiber/resin and titanium/resin were 75.53 and $97.12 \mathrm{~N}$, indicating that the bending strength of the titanium post was approximately 1.3-fold greater than that of the fiber post. This result was consistent with the findings of previous clinical studies in which the frequency of fiber post fracture was high [11] [12], but contrasted with previous findings that the modulus of elasticity of a fiber post was small and similar to that of dentin, while its bending strength was similar to that of a metal post.

The findings of a 15-year clinical study showed better outcomes with the combination of an existing metal post and resin core than with a cast metal post [6]. Furthermore, a fatigue test with bovine teeth revealed that the fracture intensity of the combination of a thin metal post and resin core was significantly greater than the fracture intensities of combinations of a cast metal post, resin post, and fiber post with resin core [26]. In a fatigue test of teeth with a thin root canal wall and damaged root canal region, a tooth with a metal post in the entire 
root canal and tooth with combination with resin around the thin metal post were compared; the results showed that fracture intensity increased by up to $50 \%$ in the combination of the thin metal post with resin [27].

In a systematic review concerning post-retained single crowns [28], a post with a high elastic modulus was recommended for teeth without ferrules. In this study, stress distribution assessed with finite element analysis was similar between the straight fiber-reinforced post with composite resin and the straight titanium post with composite resin, implying that both preparations may prevent root fracture. However, in teeth without ferrules, the titanium post with composite resin showed a high elastic modulus; this approach may be more effective because it is capable of preventing both post fracture and root fracture.

When the contour diagram prepared with the finite element analysis was compared between straight and taper types among titanium posts, stress was scattered over a wider range in the tapered titanium post than in the straight titanium post. In contrast, there was almost no difference between the straight and tapered types of fiber-reinforced post, both of which exhibit elastic modulus relatively similar to that of dentin and resin, suggesting that the use of a tapered post is more effective than a straight post for stress distribution when the elastic modulus is high.

There were a few limitations in this study. First, this study did not fully reflect actual biological conditions because the von Mises stress and gap distance were measured using finite element analysis. Second, occlusal force was only applied to one direction in each analysis. This aspect of the model did not accurately reflect actual jaw movement or occlusion. Overall, because this study was based on finite element analysis, a study of actual in vivo conditions will be necessary in the future.

\section{Conclusions}

On the basis of the findings of this finite element analysis study, the following conclusions were drawn:

1) In a tooth without a ferrule, stress distribution with the titanium straight post and resin combination was similar to that with the fiber post and resin combination; bending strength was also high, suggesting that this combination is less likely to cause root or post fracture.

2) Compared with the straight post, the combination of a titanium taper post and resin was more capable of dispersing stress in a tooth without a ferrule.

\section{Acknowledgements}

The authors are grateful to Swany Co., Ltd. (https://www.swany-ina.com/) for helping with a three-dimensional model preparation and analyzing. The authors thank Ryan Chastain-Gross, Ph.D., from Edanz (https://jp.edanz.com/ac) for editing a draft of this manuscript. 


\section{Conflicts of Interest}

The authors declare no conflicts of interest regarding the publication of this paper.

\section{References}

[1] Axelsson, P., Nyström, B. and Lindhe, J. (2004) The Long-Term Effect of a Plaque Control Program on Tooth Mortality, Caries and Periodontal Disease in Adults. Results after 30 Years of Maintenance. Journal of Clinical Periodontology, 31, 749-757. https://doi.org/10.1111/j.1600-051X.2004.00563.x

[2] Marchionatti, A.M.E., Wandscher, V.F., Rippe, M.P., Kaizer, O.B. and Valandro, L.F. (2017) Clinical Performance and Failure Modes of Pulpless Teeth Restored with Posts: A Systematic Review. Brazilian Oral Research, 31, Article No. e64. https://doi.org/10.1590/1807-3107BOR-2017.vol31.0064

[3] Soares, C.J., Valdivia, A.D.C.M., Silva, G.R.D., Santana, F.R. and Menezes, M.D.S. (2012) Longitudinal Clinical Evaluation of Post Systems: A Literature Review. Brazilian Dental Journal, 23, 135-740. https://doi.org/10.1590/S0103-64402012000200008

[4] Cagidiaco, M.C., Goracci, C., Garcia-Godoy, F. and Ferrari, M. (2008) Clinical Studies of Fiber Posts: A Literature Review. The International Journal of Prosthodontics, 21, 328-336.

[5] Pereira, J.R., do Valle, A.L., Shiratori, F.K., Ghizoni, J.S. and Bonfante, E.A. (2014) The Effect of Post Material on the Characteristic Strength of Fatigued Endodontically Treated Teeth. The Journal of Prosthetic Dentistry, 112, 1225-1230. https://doi.org/10.1016/j.prosdent.2014.03.014

[6] Hikasa, T., Matsuka, Y., Mine, A., Minakuchi, H., Hara, E.S., Van Meerbeek, B., et al. (2010) A 15-Year Clinical Comparative Study of the Cumulative Survival Rate of Cast Metal Core and Resin Core Restorations Luted with Adhesive Resin Cement. The International Journal of Prosthodontics, 23, 397-405.

[7] Stankiewicz, N.R. and Wilson, P.R. (2002) The Ferrule Effect: A Literature Review. International Endodontic Journal, 35, 575-581. https://doi.org/10.1046/j.1365-2591.2002.00557.x

[8] Magne, P., Lazari, P.C., Carvalho, M.A., Johnson, T. and Del Bel Cury, A.A. (2017) Ferrule-Effect Dominates Over Use of a Fiber Post When Restoring Endodontically Treated Incisors: An in Vitro Study. Operative Dentistry, 42, 396-406. https://doi.org/10.2341/16-243-L

[9] Ma, P.S., Nicholls, J.I., Junge, T. and Phillips, K.M. (2009) Load Fatigue of Teeth with Different Ferrule Lengths, Restored with Fiber Posts, Composite Resin Cores, and All-Ceramic Crowns. The Journal of Prosthetic Dentistry, 102, 229-234. https://doi.org/10.1016/S0022-3913(09)60159-1

[10] Ziebert, G.J. and Johnson, R.S. (1983) A Cast Dowel-Core Technique for Multirooted Teeth with Divergent Canals. The Journal of Prosthetic Dentistry, 49, 207-209. https://doi.org/10.1016/0022-3913(83)90502-4

[11] Naumann, M., Blankenstein, F. and Dietrich, T. (2005) Survival of Glass Fibre Reinforced Composite Post Restorations after 2 Years-An Observational Clinical Study. Journal of Dentistry, 33, 305-312. https://doi.org/10.1016/j.jdent.2004.09.005

[12] Naumann, M., Koelpin, M., Beuer, F. and Meyer-Lueckel, H. (2012) 10-Year Survival Evaluation for Glass-Fiber-Supported Postendodontic Restoration: A Prospective Observational Clinical Study. Journal of Endodontics, 38, 432-435. 
https://doi.org/10.1016/j.joen.2012.01.003

[13] Ko, C.C., Chu, C.S., Chung, K.H. and Lee, M.C. (1992) Effects of Posts on Dentin Stress Distribution in Pulpless Teeth. The Journal of Prosthetic Dentistry, 68, 421-427. https://doi.org/10.1016/0022-3913(92)90404-X

[14] Dejak, B. and Młotkowski, A. (2011) Finite Element Analysis of Strength and Adhesion of Cast Posts Compared to Glass Fiber-Reinforced Composite Resin Posts in Anterior Teeth. The Journal of Prosthetic Dentistry, 105, 115-126. https://doi.org/10.1016/S0022-3913(11)60011-5

[15] Lanza, A., Aversa, R., Rengo, S., Apicella, D. and Apicella, A. (2005) 3D FEA of Cemented Steel, Glass and Carbon Posts in a Maxillary Incisor. Dental Materials: Official Publication of the Academy of Dental Materials, 21, 709-715. https://doi.org/10.1016/j.dental.2004.09.010

[16] Pegoretti, A., Fambri, L., Zappini, G. and Bianchetti, M. (2002) Finite Element Analysis of a Glass Fibre Reinforced Composite Endodontic Post. Biomaterials, 23, 2667 2682. https://doi.org/10.1016/S0142-9612(01)00407-0

[17] Coelho, C.S., Biffi, J.C., Silva, G.R.D., Abrahão, A., Campos, R.E. and Soares, C.J. (2009) Finite Element Analysis of Weakened Roots Restored with Composite Resin and Posts. Dental Materials Journal, 28, 671-678.

[18] Trindade, F.Z., Valandro, L.F., de Jager, N., Bottino, M.A. and Kleverlaan, C.J. (2018) Elastic Properties of Lithium Disilicate Versus Feldspathic Inlays: Effect on the bonding by 3D Finite Element Analysis. Journal of Prosthodontics: Official Journal of the American College of Prosthodontists, 27, 741-747.

https://doi.org/10.1111/jopr.12550

[19] Chieruzzi, M., Pagano, S., Pennacchi, M., Lombardo, G., D’Errico, P. and Kenny, J.M. (2012) Compressive and Flexural Behaviour of Fibre Reinforced Endodontic Posts. Journal of Dentistry, 40, 968-978. https://doi.org/10.1016/j.jdent.2012.08.003

[20] Lo Giudice, G., Ferrari Cagidiaco, E., Lo Giudice, R., Puleio, F., Nicita, F. and Calapaj, M. (2019) Evaluation of Mechanical Properties of a Hollow Endodontic Post by Three Point Test and SEM Analysis: A Pilot Study. Materials, 12, Article No. 1983. https://doi.org/10.3390/ma12121983

[21] Asmussen, E., Peutzfeldt, A. and Sahafi, A. (2005) Finite Element Analysis of Stresses in Endodontically Treated, Dowel-Restored Teeth. The Journal of Prosthetic Dentistry, 94, 321-329. https://doi.org/10.1016/j.prosdent.2005.07.003

[22] Nokar, S., Bahrami, M. and Mostafavi, A.S. (2018) Comparative Evaluation of the Effect of Different Post and Core Materials on Stress Distribution in Radicular Dentin by Three-Dimensional Finite Element Analysis. Journal of Dentistry (Tehran), 15, 69-78.

[23] Rodríguez-Cervantes, P.J., Sancho-Bru, J.L., González-Lluch, C., Pérez-González, A., Barjau-Escribano, A. and Forner-Navarro, L. (2011) Premolars Restored with Posts of Different Materials: Fatigue Analysis. Dental Materials Journal, 30, 881-886.

[24] Cuadros-Sanchez, J., Szesz, A., Hass, V., Patzlaff, R.T., Reis, A. and Loguercio, A.D. (2014) Effects of Sonic Application of Adhesive Systems on Bonding Fiber Posts to Root Canals. Journal of Endodontics, 40, 1201-1205. https://doi.org/10.1016/j.joen.2013.12.034

[25] Dietschi, D., Duc, O., Krejci, I. and Sadan, A. (2008) Biomechanical Considerations for the Restoration of Endodontically Treated Teeth: A Systematic Review of the Literature, Part II (Evaluation of Fatigue Behavior, Interfaces, and in Vivo Studies). Quintessence International, 39, 117-129.

[26] Fukui, Y., Komada, W., Yoshida, K., Otake, S., Okada, D. and Miura, H. (2009) Ef- 
fect of Reinforcement with Resin Composite on Fracture Strength of Structurally Compromised Roots. Dental Materials Journal, 28, 602-609.

[27] Saupe, W.A., Gluskin, A.H. and Radke Jr., R.A., (1996) A Comparative Study of Fracture Resistance Between Morphologic Dowel and Cores and a Resin-Reinforced Dowel System in the Intraradicular Restoration of Structurally Compromised Roots. Quintessence International, 27, 483-491.

[28] Sarkis-Onofre, R., Fergusson, D., Cenci, M.S., Moher, D. and Pereira-Cenci, T. (2017) Performance of Post-Retained Single Crowns: A Systematic Review of Related Risk Factors. Journal of Endodontics, 43, 175-183.

https://doi.org/10.1016/j.joen.2016.10.025 